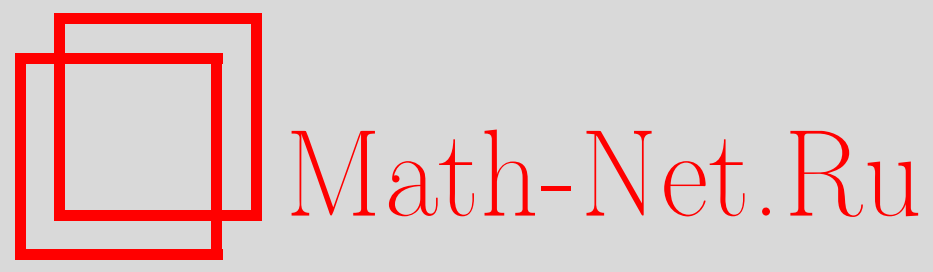

А. М. Степаненков, О сложности проверки простоты числа однородными структурами, Дискрет. матем., 2003, том 15, выпуск $3,54-65$

DOI: https://doi.org/10.4213/dm205

Использование Общероссийского математического портала Math-Net.Ru подразумевает, что вы прочитали и согласны с пользовательским соглашением http: //www . mathnet.ru/rus/agreement

Параметры загрузки:

IP : 34.227 .88 .159

26 апреля 2023 г., 09:14:24

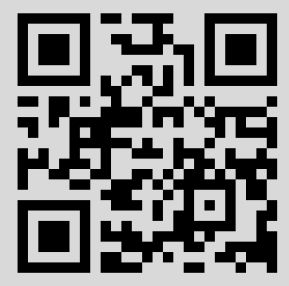


УдК 519.7

\title{
О сложности проверки простоты числа однородными структурами
}

\author{
(c) 2003 г. А. М. Степаненков
}

\begin{abstract}
В статье показано, что при Тьюринговой кодировке натуральных чисел их простота проверяется однородными структурами за время, асимптотически совпадающее с половиной длины кода.

Работа выполнена при поддержке Российского фонда фундаментальных исследований, проект 02-01-00162.
\end{abstract}

\section{1. Введение}

Проверка свойств натуральных чисел важна в приложениях теории чисел. Для обнаружения этих свойств строятся алгоритмы, сложность которых измеряет трудность проверки выполнимости этих свойств.

Одной из перспективных моделей алгоритмов являются однородные структуры автоматов, поскольку наряду с наглядностью представления данных и простотой задания самой однородной структуры в них реализуется процесс параллельной обработки информации. Последнее свойство и дает возможность проверить свойство простоты за асимптотически линейное время.

\section{2. Основные понятия и результаты}

Введем понятие однородной структуры.

Содержательно, однородная структура (OC) представляет собой бесконечную схему, построенную из копий одного и того же конечного автомата, и такую, что правила соединения входов произвольного автомата с выходами других автоматов в ней везде одинаковы. В дальнейшем эти автоматы будем называть ячейками ОС. Более формально, однородной структурой размерности $k$ называется четверка $\left(Z^{k}, Q, V, \varphi\right)$, где $Z^{k}$ есть множество $k$-мерных векторов с целыми координатами, $Q-$ конечное множество состояний, $V=\left\{\alpha_{1}, \ldots, \alpha_{n-1}\right\}-$ упорядоченный набор различных ненулевых векторов из $Z^{k}$, называемый шаблоном соседства ОС и определяющий для каждой ячейки $\alpha$ ее окрестность

$$
V(\alpha)=\left\{\alpha, \alpha+\alpha_{1}, \ldots, \alpha+\alpha_{n-1}\right\},
$$

$\varphi: Q^{n} \rightarrow Q$ - локальная функция переходов. Если в момент времени $t$ состояния ячеек $\alpha, \alpha+\alpha_{1}, \ldots, \alpha+\alpha_{n-1}$ были равны соответственно $x_{0}, x_{1}, \ldots, x_{n-1}$, то состояние $\alpha$ в момент $t+1$ полагается равным $\varphi\left(x_{0}, x_{1}, \ldots, x_{n-1}\right)$. Состоянием ОС будем называть 
функщию, сопоставляющую каждой ячейке ее состояние из множества $Q$. Если состояние ОС в момент времени $t$ есть $f$, то ее состояние в момент $t+1$ есть функщия $g$, определяемая равенством

$$
g(\alpha)=\varphi\left(f(\alpha), f\left(\alpha+\alpha_{1}\right), \ldots, f\left(\alpha+\alpha_{n-1}\right)\right) .
$$

Это равенство задает основную функцию переходов $\Phi: g=\Phi(f)$. Поведением ОС назовем последовательность $\left\{f_{i}\right\}$ ее состояний такую, что $f_{i+1}=\Phi\left(f_{i}\right)$. В дальнейшем поведение однородной структуры часто будем называть функционированием ОС.

Сушествует выделенный элемент множества $Q$ - символ пустоты. Будем обозначать его через П. Далее рассматриваем лишь такие ОС, у которых $\varphi(\Pi, \ldots, \Pi)=\Pi$. Состояние П является состоянием покоя. Состояние ОС, у которого лишь конечное число ячеек находится в отличном от П состоянии, назовем конфигурацией.

Перейдем к формальной постановке задачи о проверке простоты натурального числа с помощью однородных структур. Под кодом натурального числа $N$ будем понимать цепочку, состоящую из $N$ одинаковых символов. Требуется построить плоскую однородную структуру такую, что если код любого натурального числа $N>1$ записать в ее ячейки (то есть, привести цепочку из $N$ автоматов в состояние, соответствующее символу кода; остальные автоматы должны при этом находиться в выделенном (пустом) состоянии), то через конечное число шагов в результате функщионирования ОС возникнет следуюшая конфигурашия: все ячейки, кроме одной, пусты, а последняя пребывает в одном из состояний $P$ или $C$. Причем во все последующие моменты времени состояние ОС не изменяется. Это состояние ОС будем интерпретировать как ответ на вопрос, простое ли число $N$. Если ОС дает правильный ответ для любого натурального числа, то говорят, что данная ОС решает задачу проверки простоты натурального числа.

Введем некоторые ограничения на ОС. Число состояний ячейки должно быть ограничено некоторой константой $m$, то есть $|Q| \leqslant m$. В качестве шаблона соседства может выступать любой упорядоченный набор, состоящий из элементов множества

$$
V=\{(-1,-1),(0,-1),(1,-1),(-1,0),(1,0),(-1,1),(0,1),(1,1)\}
$$

Естественно стараться оптимизировать время работы ОС. Будем выражать его как функцию длины $N$ задачи (длины $N$ кода числа $N$ ). Используем для времени работы ОС $\sigma$ обозначение $T_{\sigma}(N)$.

Далее будем рассматривать только те ОС, которые удовлетворяют этим ограничениям. Рассмотрим все ОС, которые решают задачу о проверке простоты натурального числа, то есть, дают ответ $P$, если число простое, и $C$, если оно составное.

Введем функцию сложности

$$
T(N)=\min \left\{T_{\sigma}(N): \sigma \text { решает задачу о проверке числа на простоту }\right\}
$$

Основным результатом работы является следующее утверждение.

Теорема 1. При $N \rightarrow \infty$

$$
T(N) \sim N / 2
$$




\section{3. Нижняя оценка для $T(N)$}

Лемма 1. Для любого $N>1$

$$
T(N) \geqslant[N / 2]-1
$$

Доказательство. Предположим, что существует $N$ такое, что $T(N)<[N / 2]-1$. Тогда из определения функции $T$ следует, что сушествует ОС $\sigma$, которая проверяет простоту $N$ за время, меньшее [N/2] - 1. Обозначим ее время работы $K$.

Под окрестностью ячейки $\alpha$ размера $k$ будем понимать множество ячеек таких, что сигнал от них может достигнуть $\alpha$ за $k$ или менее тактов. Скажем, что окрестность ячейки $\alpha_{1}$ размера $k$ в момент времени $t_{1}$ совпадает с окрестностью ячейки $\alpha_{2}$ размера $k$ в момент времени $t_{2}$, если конфигурация окрестности ячейки $\alpha_{1}$ в момент времени $t_{1}$ совпадает с конфигурацией ячейки $\alpha_{2}$ в момент времени $t_{2}$. Заметим, что эти понятия зависят от шаблона соседства.

Поскольку время работы ОС $\sigma$ на числе $N$ строго меньше [N/2] -1 , существуют символы кода, которые не попадают в окрестность ответа размера $K$. Однако в окрестность ответа обязательно должен входить либо крайний левый, либо крайний правый символ кода. Действительно, если это не так, то в силу того, что $K<[N / 2]-1$, существует ячейка, окрестность размера $K$ которой в начальный момент времени совпадает с окрестностью ответа того же размера в тот же момент времени. Следовательно, через $K$ тактов она перейдет не в пустое состояние, а либо в $P$, либо в $C$, что недопустимо. Покажем, что на всех числах $M$, больших $N$, ОС $\sigma$ будет выдавать тот же ответ, что и на $N$.

Конфигурация для числа $M$ получается из конфигурации для числа $N$ приписыванием некоторого числа символов кода. Будем считать, что все символы кода приписываются так, что они не попадают в окрестность ячейки размера $K$, в которой был зафиксирован ответ. Рассмотрим ячейку, в которой был сформирован ответ при обработке числа $N$. Очевидно, ее окрестность размера $K$ в начальный момент времени не изменилась. Следовательно, через $K$ тактов эта ячейка перейдет в то же самое состояние, то есть возникнет тот же ответ.

Теперь рассмотрим любую другую ячейку. Покажем, что через $K$ тактов она перейдет в пустое состояние. Тем самым мы докажем, что на любом числе $M$, большем $N$, однородная структура $\sigma$ выдает тот же ответ, что и на $N$. Справедливость сформулированного только что утверждения следует из того, что для любой подобной ячейки $\alpha$ существует ячейка $\beta$ такая, что окрестность ячейки $\alpha$ размера $K$ в новой задаче в начальный момент времени совпадает с окрестностью ячейки $\beta$ размера $K$ в старой задаче в начальный момент времени. Действительно, ячейки $\alpha$ можно разделить на два типа: ячейки, в окрестность размера $K$ которых попадает хотя бы один крайний символ кода, и остальные ячейки (они существуют в силу того, что $K<[N / 2]-1$ и ячейка с ответом не обладает этим свойством). Ясно, что окрестности размера $K$ ячеек второго типа с одинаковыми ординатами в начальный момент времени равны. Приписывание же новых символов кода на самом деле порождает ячейки второго типа, так как $K<[N / 2]-1$.

Таким образом, если $N$ было простым, то возьмем составное $M=N+1$. (Ясно, что $N$ не равно 2 , так как тогда $K<0$.) Если же $N$ было составным, то будем прибавлять к нему по единице, пока не получим простое число $M$. В обоих случаях ОС $\sigma$ дает одинаковый ответ на числах $N$ и $M$, что противоречит определению ОС $\sigma$, как решающей задачу о проверке простоты натурального числа. Лемма доказана. 


\section{4. Верхняя оценка для $T(N)$. Асимптотически наилучший алгоритм проверки простоты числа}

Сначала дадим общее описание алгоритма.

Лемма о нижней оценке по существу дает подсказку, как надо действовать для того, чтобы получить асимптотически наилучший алгоритм: надо собирать информацию в центре конфигурации. И сразу же после того, как последний сигнал добрался до этого центра, или спустя некоторое время $o(N)$ на основе собранных данных делать вывод. Так мы и будем действовать. Со стороны функционирование ОС будет выглядеть так: конфигурация, состояшая из палочек, будет стремительно уменьшаться в размерах с обоих концов кода. Однако, во-первых, информация не должна теряться, во-вторых, информация (палочная кодировка числа) должна воплотиться в нечто более компактное, анализ чего не составил бы труда, например, в двоичное представление числа. Поэтому, сворачиваясь, конфигурация будет преобразовывать себя в двоичный код натурального числа $N$. Когда мы его получим, все преобразования, которые будут вестись, будут преобразованиями над данными длины $\log N$, а это значит, что нам позволяется применять практически любые алгоритмы, в том числе переборные, и все равно мы остаемся в условиях ограничения функцией вида $o(N)$.

Вначале рассмотрим вспомогательную задачу проверки простоты натурального числа в плоских однородных структурах для случая, когда кодировка представляет собой его двоичное представление. Для этого случая справедлива следующая лемма.

Јемма 2. Существует плоская однородная структура, решающая задачу определения простоты натурального числа $N$ при двоичной кодировке за время $о(N)$ при $N \rightarrow \infty$.

Доказательство. Утверждение леммы 2 вытекает из того, что можно предложить простой алгоритм, построить машину Тьюринга, которая решала бы поставленную задачу. Предположим, что алфавит машины Тьюринга есть множества пар $(a, b)$, где $a$ и $b$ могут быть $\Pi, 0,1, P, C$ и некоторыми другими вспомогательными символами. Начальная конфигурация на ленте - это двоичный код числа, записанный в первых компонентах вектора, во вторых компонентах - символы пустоты. Головка стоит над первым символом кода. Финальная конфигурация: на ленте существует лишь один символ $(a, b)$, отличный от $(\Pi, \Pi)$. Он имеет вид $(P, \Pi)$ или $(C, \Pi)$, и над ним стоит головка машины Тьюринга. В дальнейшем, мы будем часто использовать в качестве алфавита состояний конечное множество векторов некоторой размерности, так как всегда можно построить взаимно однозначное соответствие между алфавитом векторов-состояний и классическим алфавитом состояний, использование векторов-состояний более удобно для описания алгоритмов. Координаты вектора-ячейки однородной структуры или вектора-ячейки ленты машины Тьюринга будем называть слоями.

Алгоритм состоит в следующем.

Шаг 1. Формирование во втором слое двоичного кода числа 1 , начало которого совпадает с началом двоичного кода числа $N$. Переход на начало кода числа $N$. Это можно сделать за константное время.

Шаг 2. Пусть длина кода числа, записанного в первом слое, есть $n$, во втором $k$. Проверка того, что $n>2 k-1$. Переход на начало кода числа в первом слое, но в различных состояниях, в зависимости от результата сравнения. Это можно сделать 
за квадратичное время (нанести во втором слое справа от кода числа его копию - квадратичное время, проверить неравенство $n>2 k-1$ - константное время, стереть копию - линейное время).

Шаг 3. Если $n>2 k-1$, то число $N$ простое. Надо стереть его код и код числа во втором слое. Написать $(P, \Pi)$, остановиться и перейти в заключительное состояние. В противном случае, прибавить 1 к числу, записанному во втором слое. Установить головку на начало кода числа в первом слое. Обе операции выполняются за линейное время.

Шаг 4. Скопировать коды чисел первого и второго слоя вправо и остановиться на их начале, на что требуется квадратичное время.

Шаг 5. Проверка того, что число в первом слое делится на число во втором слое на основе алгоритма деления в столбик. Стирание копий. Установка головки на начало первоначального кода числа $N$ и переход в состояние, соответствующее результату проверки, на что требуется квадратичное время.

Шаг 6. Если результат проверки положительный, то $N$ составное. Надо стереть его код и код числа во втором слое. Написать $(C, \Pi)$, остановиться и перейти в заключительное состояние. В противном случае переход к шагу 2.

Легко видеть, что число итераций в шагах 2-6 есть $O\left(2^{n / 2}\right)$. Время, затрачиваемое на каждой итерации, есть $O\left(n^{2}\right)$, так что общее время есть $O\left(n^{2} 2^{n / 2}\right)=o(N)$. В свою очередь, любую машину Тьюринга легко смоделировать с помощью однородной структуры размерности $k$ с шаблоном соседства $\{(-1,0, \ldots, 0),(1,0, \ldots, 0)\}$ без замедления следующим образом.

Рассмотрим машину Тьюринга с входным алфавитом $A$, алфавитом состояний $Q$, тогда моделирующая ОС может выглядеть следующим образом: алфавит состояний $A \times\{0,1\} \times Q$, то есть состояние ячейки ОС складывается из символа алфавита $A$ на ленте машины Тьюринга, символа отсутствия или присутствия головки из алфавит 0,1 и (в случае присутствия головки) символа состояния машины Тьюринга из алфавит $Q$. Функция переходов выглядит следующим образом. Это тождественная функцигя, если в окрестности ячейки нет ячейки в состоянии $(a, 1, c)$, где $a \in A, c \in Q$ произвольны. В остальных случаях она строится по программе машины Тьюринга.

Из описанного выше алгоритма и возможности моделировать машину Тыюринга в однородных структурах вытекает справедливость утверждения леммы 2.

Рассмотрим ОС $\sigma_{1}$, которая проверяет простоту любого натурального числа, большего тройки, со временем работы меньшим $2^{|n / 2|}+n^{2} / 2+5 n+7$. ОС $\sigma_{1}$ имеет шаблон соседства

$$
V_{2}=\{(-1,-1),(0,-1),(1,-1),(-1,0),(1,0),(0,1)\}
$$

Сама ячейка представляет собой шестимерный вектор. Координаты (слои) могут принимать следующие состояния:

1-й слой принимает состояния $\Pi, 1,0$;

2-й $-\Pi, 1,0,<, \#$;

3-й - П, P, C, U, D, B, $c, X, x, y, m, u, v, l, r, o,<,>, \#, /, \mid, !, ?, 9,8,7,6,5,4,1,0$; 


$$
\begin{aligned}
& \text { 4-й }-\Pi, 1, p ; \\
& \text { 5-й }-\Pi, 1,0, s ; \\
& \text { 6-й }-\Pi, 1,0 .
\end{aligned}
$$

Как видно, число состояний, которые может принимать ячейка $\mathrm{OC} \sigma_{1}$, не превышает $3 \cdot 5 \cdot 31 \cdot 3 \cdot 4 \cdot 3=16740$.

Начальной конфигурацией для ОС $\sigma_{1}$ будет цепочка двоичного кода числа $N$, записанная в первом слое. Также в третий слой второй (от начала кода) ячейки наносится символ $>$, а в тот же самый слой последней - символ $<$.

Функционирование ОС $\sigma_{1}$ можно условно разбить на четыре процесса.

Первый процесс производит проверку числа на четность и (в случае отрицательного результата) производит подготовку к размножению вниз кода числа, записанного в первом слое, и кодов нечетных потенциальных делителей, записанных во втором слое. Поскольку при проверке на простоту число $N$ достаточно делить только на простые, меньшие ]$\sqrt{N}[+1$, то, отступая на одну ячейку от середины кода числа в третьем слое, формируется сигнал $x$, который размножается вместе с потенциальными делителями. Таким образом, можно остановить процесс размножения после того, как появится делитель равный $] \sqrt{N}$ [ (точнее, $2^{\text {]n/2[ }}-1$ ). После этого запускается процесс остановки и уничтожения размножающейся вниз части конфигурации. Уничтожающие сигналы могут возникнуть и при появлении в третьем слое сигнала $C$. Параллельно с размножением, в каждой строке, где нанесен код числа $N$ в первом слое и код его потенциального делителя $K$ во втором, осуществляется процесс проверки делимости $N$ на $K$ (на основе алгоритма деления в столбик). Результат проверки в виде сигналов $P$ или $C$ записывается в третьем слое, лишняя информация стирается, а вышеупомянутые сигналы передаются вниз, где на их основе формируется окончательный ответ.

Опишем подробнее каждый блок.

Блок 1. Подготовка к размножению. Итак, первое, что делается, это проверка числа $N$ на четность (если в последней непустой ячейке в первом слое стоит символ 0 , то $N$ четно, и мы даем ответ $C$ ). В случае отрицательного результата проверки, сигналы > и < двигаются навстречу друг другу для того, чтобы обозначить то место на числе $N$, до которого позволительно расти потенциальным делителям $K$. Далее синхронизируется рост части конфигурации, связанной с формированием делителей $K$, и движение впоследствии останавливающего этот рост сигнала $x$. На этапе подготовки затрачивается время, которое не превышает $3[(n+1) / 2]+1$.

Блок 2. Размножение. Размножение кода числа $N$ и формирование кодов его потенциальных делителей $K$ происходит с задержкой в один такт. Сделано это для того, чтобы в случае возникновения сигнала $C$, уничтожающие сигналы сумели догнать разрастающуюся вниз конфигурацию. Передача вниз кода числа $N$ происходит довольно просто: он с задержкой в один такт копируется вниз в первый слой. Формирование кодов потенциальных делителей $K$ происходит несколько сложнее. За это отвечают третий, пятый и шестой слои. Новый потенциальный делитель формируется во втором слое на основе прибавления двойки к потенциальному делителю $K-2$, записанного во втором слое верхних ячеек. (Заметим, что на этапе подготовки формируется код первого потенциального делителя - тройки.) 
Блок 3. Проверка на кратность одного натурального числа другому. Здесь решается следуюшая задача: делится ли число $N$, записанное в первом слое ячеек, на число $K$, записанное во втором слое ячеек непосредственно под числом $N$.

В первую очередь проверяется корректность записи чисел $N$ и $K$. А именно, если число $K$ больше соответствующей части числа $N$ (то есть начала числа $N$, под которым записано число $K$ ), то его необходимо сдвинуть на одну ячейку вправо. Затем, осуществляем вычитание $N-K$ с последующим сдвигом числа $K$ вправо, и снова все повторяется заново. Выполнение цикла осуществляется до тех пор, пока правая часть кода $K$ не совмещается с правой частью кода $N$. В случае тождественного совпадения кода $K$ и кода остатка $N$ мы делаем вывод, что $K$ делит $N$, в противном случае $K$ не делит $N$. В первом случае в третьем слое ячейки ставим символ $C$ (число $N$ не простое), во втором случае ставим символ $P$ (число $N$ после деления на $K$ сохраняет возможность быть простым). В заключение, очищаем все ячейки (кроме той, которая содержит ответ). Время проверки на кратность числа $N$ числу $K$, если считать размеры их кодов равными $n$ и $k$, соответственно, не больше $2 k(n-k)+n+1$ тактов.

Блок 4. Остановка роста конфигурации. Удаление лишней информации. Критерий остановки роста конфигурации следующий: либо в процессе проверки на кратность выяснилось, что $N$ составное, либо закончились потенциальные делители, то есть $N$ простое. В первом случае формируется сигнал $C$, который поглощает сигналы $P$, во втором случае имеют место только сигналы $P$, которые сольются в один. В обоих случаях возникает уничтожающий сигнал со скоростью движения, в два раза превышающей скорость роста конфигурации, который обеспечивает уничтожение всех сигналов, отличных от $C$ и $P$.

Как видно, худший случай времени работы ОС $\sigma_{1}$ достигается на простых числах. В случае составного $N$ время работы ОС $\sigma_{1}$ асимптотически равно наименьшему делителю числа $N$. В приведенной однородной структуре на каждом такте задействуется не так много автоматов-вычислителей - размер максимальной конфигурации не больше $n^{3} / 4+5 n^{2} / 4+2 n$. Путем вовлечения в работу большего числа вычислителей можно сушественно улучшить время работы в худшем случае. Сделать это можно следующим образом.

Будем проверять делимость числа $N$ на блоках, состоящих из $O\left(2^{n / 4}\right)$ потенциальных делителей. Проверка на делимость в разных блоках будет осуществляться параллельно.

Сначала осушествляем проверку на четность.

Затем сформируем начальную конфигурацию первого блока. Она будет состоять из кода числа $N$, кода первого потенциального делителя, тройки, и кода четного числаограничителя порядка $2^{n / 4}$, а также дополнителіных символов, необходимых алгоритму.

На каждом блоке применяется уже описанный алгоритм, у которого изменится лишь критерий возникновения уничтожающего сигнала. Появляться он будет тогда и только тогда, когда очередной потенциальный делитель превысит число-ограничитель. Следовательно, время работы нового алгоритма на таком блоке есть $O\left(2^{n / 4}\right)$.

Начальная конфигурация каждого блока один раз копируется вправо следуюшим образом: копируется код числа $N$, формируется код первого потенциального делителя, равного увеличенному на единицу числу-ограничителю соседнего слева блока, формируется код числа ограничителя, равный сумме числа-ограничителя первого блока и числаограничителя соседнего слева блока. Все эти действия можно осуществить за время $O(n)$. 
Критерий остановки роста конфигурации вправо - первый потенциальный делитель превысил $2^{\mid n / 2 I}$.

В результате проверки на делимость в каждом блоке формируется свой промежуточный результат. Эти результаты необходимо объединить. Ответ каждого блока, кроме первого будет направляться влево, где он объединяется с ответом соседнего блока.

Нетрудно оценить время работы этого алгоритма. Оно складывается из времени формирования начальной конфигурации последнего блока, которое есть $O\left(n 2^{n / 4}\right)$, времени проверки на делимость в последнем блоке, которое есть $O\left(2^{n / 4}\right)$ и времени передачи промежуточного результата в первый блок, которое есть $O\left(n 2^{n / 4}\right)$. В сумме получится $O\left(n 2^{n / 4}\right)$. Несложно посчитать максимальное число автоматов-вычислителей, задействованных в работе. Максимальное число непустых ячеек в каждом блоке есть $O\left(n^{3}\right)$. Поскольку новые блоки формируются с задержкой в $O(n)$, число блоков, в которых ведутся вычисления, есть $O\left(2^{n / 4} / n\right)$. В итоге получаем, что размер максимальной конфигурации есть $O\left(n^{2} 2^{n / 4}\right)$. Таким образом, добавив $O\left(2^{n / 4} / n\right)$ новых вычислителей, мы во столько же уменьшили верхнюю оценку времени работы ОС.

Перейдем к заключительному шагу.

Лемма 3. Существует плоская однородная структура, решающая задачу проверки простоты натурального числа $N$, заданного в палочной кодировке за время $N / 2+o(N)$ при $N \rightarrow \infty$.

Доказательство. В качестве базовой возьмем ОС $\sigma_{1}$, которая решает задачу простоты любого натурального числа, большего тройки, представленного в двоичном коде. Добавив новые состояния ячейки и правила перехода, получим новую однородную структуру $\sigma_{2}$, решающую задачу простоты натурального числа $N$, палочный код которой записан во втором слое. Если кратко, то функционирование ОС $\sigma_{2}$ будет состоять из двух этапов: на первом конфигурация, состоящая из палочного представления, преобразуется в начальную конфигурацию для ОС $\sigma_{1}$, и обрабатываются случаи для $N=2,3$, на втором этапе ОС $\sigma_{2}$ работает в соответствие с алгоритмом для ОС $\sigma_{1}$.

Перейдем к подробному описанию процесса перекодировки натурального числа из палочного представления в двоичное. Состояния слоев ячейки будут обозначаться латинскими буквами $a, b, c, d, e, f$. Координаты ячейки будут записываться в скобках. Например, состояние второго слоя ячейки ОС с координатами $(-1,1)$ будет обозначаться $b(-1,1)$. Состояние слоя ячейки в следующий (относительно некоторого текущего) момент времени будет обозначаться латинской буквой со штрихом. Литералом П обозначается пустой символ.

Образование стартовой конфигурации. В начальный момент времени мы из палочного кода числа $N$, записанного во втором слое, образуем стартовую конфигурацию: все палочки заменяются единицами, а в первом слое крайних слева и справа ячеек генерируются сигналы > и < соответственно.

Если $b(x-1, y)=\Pi, b(x, y)=1$, то $a^{\prime}(x, y)=>$.

Если $b(x, y)=\mid, b(x+1, y)=\Pi$, то $a^{\prime}(x, y)=<$.

Если $b(x, y)=1$, то $b^{\prime}(x, y)=1$.

Правила свертки кода слева. Сигнал > движется вправо навстречу сигналу <, уничтожая по пути своего следования единицы.

Если $a(x-1, y)=>, a(x, y) \neq<, a(x+1, y) \neq<$, то $a^{\prime}(x, y)=>$.

Если $a(x, y)=>, b(x, y)=1$, то $a^{\prime}(x, y)=\Pi, b^{\prime}(x, y)=\Pi$. 
Правила свертки кода справа. Сигнал < движется влево до встречи с >, реализуя в процессе движения формирование двоичного кода числа, равного сумме поглошаемых им единиц.

Если $a(x-1, y) \neq>, a(x, y) \neq>, a(x+1, y)=<$, то $a^{\prime}(x, y)=<$.

Если $a(x, y)=<$, то $a^{\prime}(x, y)=\Pi$.

Двоичный код будет записываться во втором слое справа налево, то есть, левее всех будет младший разряд. Формирование младшего разряда описано ниже.

Если $a(x+1, y)=<, b(x+1, y)=0$, то $b^{\prime}(x, y)=1$.

Если $a(x+1, y)=<, b(x+1, y)=1$, то $b^{\prime}(x, y)=0$.

Двоичный код в процессе формирования движется вслед за своим создателем - сигналом <, изменяясь в пути. Это движение обеспечивают сигналы / шестого слоя. Их формированием также занимается сигнал <. В процессе прибавлений единиц необходимо иногда держать единицу в уме для прибавления ее к старшему разряду. Это обеспечивает символ 1 , который стоит в пятом слое и меняет пробегающие мимо во втором слое 1 на 0,0 на 1 до тех пор, пока не произойдет прибавка к старшему разряду (то есть, пока во втором слое не появится 0).

Ниже приведены правила формирования и движения сигнала /, 0 или 1 второго слоя и 1 пятого.

Если $a(x-1, y) \neq>, a(x, y)=<, b(x, y)=1, b(x+1, y) \neq \Pi$, то $b^{\prime}(x, y)=b(x+1, y)$, $e^{\prime}(x, y)=1, f^{\prime}(x, y)=/$.

Если $a(x-1, y) \neq>, a(x, y)=<, b(x, y)=1, b(x+1, y)=\Pi$, то $b^{\prime}(x, y)=b(x+1, y)$, $e^{\prime}(x, y)=1$.

Если $a(x-1, y) \neq>, a(x, y)=<, b(x, y)=0$, то $b^{\prime}(x, y)=b(x+1, y), f^{\prime}(x, y)=/$.

Если $b(x, y)=1$ или $b(x, y)=0, f(x, y)=/$, то $b^{\prime}(x, y)=b(x+1, y), f^{\prime}(x, y)=$ $f(x+1, y)$.

Далее даны условия пропадания символа 1 пятого слоя.

Если $f(x-1, y)=/, b(x, y)=0$ или $b(x, y)=\Pi, e(x, y)=1$, то $e^{\prime}(x, y)=\Pi$.

Если $a(x-1, y)=<, b(x, y)=0$ или $b(x, y)=\Pi, e(x, y)=1$, то $e^{\prime}(x, y)=\Pi$.

А это описание функщионирования символа 1 пятого слоя.

Если $a(x, y)=<, b(x+1, y)=0$ или $b(x+1, y)=\Pi, e(x+1, y)=1$, то $b^{\prime}(x, y)=1$, $f^{\prime}(x, y)=1$.

Если $a(x, y)=<, b(x+1, y)=1, e(x+1, y)=1$, то $b^{\prime}(x, y)=0, f^{\prime}(x, y)=/$.

Если $e(x, y) \neq \#, f(x, y)=/, b(x+1, y)=0$ или $b(x+1, y)=\Pi, e(x+1, y)=1$, то $b^{\prime}(x, y)=1, f^{\prime}(x, y)=1$.

Если $e(x, y) \neq \#, f(x, y)=/, b(x+1, y)=1, e(x+1, y)=1$, то $b^{\prime}(x, y)=0$, $f^{\prime}(x, y)=/$.

Здесь появился новый символ \#. Он возникает при столкновении сигналов $>$ и <. Условие неравенства ему объясняется так: в случае нечетного $N$ мы не хотим прибавлять последнюю единицу (так как в двоичной записи проще умножить число на 2 и прибавить единицу, что эквивалентно приписыванию к правой части его кода символа 1 , чем умножить на 2 и вычесть 1). Следующая строчка из той же серии означает, что вопреки общему правилу младший разряд кода передается без изменений.

Если $a(x-1, y)=>, a(x, y)=\Pi, a(x+1, y)=<$, то $b^{\prime}(x, y)=b(x+1, y), e^{\prime}(x, y)=\#$, $f^{\prime}(x, y)=/$.

Также необходимо уничтожить сформировавшуюся, возможно, на последнем шаге прибавления единицу в пятом слое.

Если $e(x-1, y)=\#, e(x, y)=1$, то $e^{\prime}(x, y)=\Pi$. 
Обработка случая четного $N$. Если сигналы > и < вплотную подошли друг к другу, то можно сделать вывод, что $N$ - четное число. В этом случае генерируется сигнал ответа $C$. Далее он передается вправо до тех пор, пока не выйдет на край.

Если $a(x-1, y)=>, a(x, y)=<$, то $a^{\prime}(x, y)=\Pi, b^{\prime}(x, y)=\Pi, c^{\prime}(x, y)=C$, $d^{\prime}(x, y)=\Pi, e^{\prime}(x, y)=\Pi, f^{\prime}(x, y)=\Pi$.

Если $c(x-1, y)=C, a(x, y) \neq \Pi$, или $b(x, y) \neq \Pi$, или $e(x, y) \neq \Pi$, или $f(x, y) \neq \Pi$, то $a^{\prime}(x, y)=\Pi, b^{\prime}(x, y)=\Pi, c^{\prime}(x, y)=c(x-1, y), e^{\prime}(x, y)=\Pi, f^{\prime}(x, y)=\Pi$.

Разворот кода. В нечетном же случае в пятом слое (как мы уже видели) рождается сигнал \# и процесс формирования кода вступает в новую фазу. Далее нам надо перекопировать бегущие по второму слою символы кода в первый слой, причем, записывая их в обратном порядке, чтобы в полученном коде крайним левым был старший разряд. Процессом копировки кода в первый слой будет заниматься символ \#. Далее нам надо разрешить символам кода забегать в ячейку с сигналом \#.

Если $e(x, y)=\#, c(x+1, y) \neq>$, то $b^{\prime}(x, y)=b(x+1, y), f^{\prime}(x, y)=f(x+1, y)$.

Сигнал \# копирует содержимое второго слоя в первый, после чего передается влево для очередного копирования.

Если $a(x, y)=\Pi, b(x, y)=1$ или $b(x, y)=0, e(x, y)=\#$, то $a^{\prime}(x, y)=b(x, y)$.

Если $b(x, y)=1$ или $b(x, y)=0, e(x, y)=\#$, то $e^{\prime}(x, y)=\Pi$.

Если $b(x+1, y) \neq \Pi, e(x+1, y)=\#$, то $e^{\prime}(x, y)=\#$.

Формирование начальной конфигурации для ОС $\sigma_{1}$. Когда перестанут поступать сигналы из второго слоя, наступит новый этап в формировании двоичного кода числа $N$. Нам надо дописать справа единицу, а также в третьем слое крайней правой ячейки сгенерировать сигнал <, в третьем слое второй слева ячейки сгенерировать сигнал >, причем сделать это надо синхронно. Тогда мы получим в точности начальную конфигурацию для ОС $\sigma_{1}$ (к чему мы и стремимся). Для реализации этого мы запустим с левого края на правый два сигнала: в третьем слое > и в пятом слое $r$, который будет двигаться с задержкой в один такт. Задержка будет организована с помощью сигнала $R$.

Если $a(x, y)=\Pi, b(x, y)=1$ или $b(x, y)=0, e(x, y)=\#, b(x+1, y)=\Pi$, то $c^{\prime}(x, y)=>, e^{\prime}(x, y)=r$.

Выполнивший свою часть работы сигнал \# стирается.

Если $e(x, y)=\#, c(x+1, y)=>$, то $e^{\prime}(x, y)=\Pi$.

Подойдя к правому краю кода, сигнал $>$ добавляет к нему единицу.

Если $c(x-1, y)=>, a(x, y)=\Pi$, то $a^{\prime}(x, y)=1$.

Остается пустить какой-то сигнал влево, который поставит на левом краю символ $>$, причем, сделает это в тот момент, когда на правом краю появится сигнал $r$. В следующий момент времени сигнал $r$ поставит в третий слой ячейки символ $<$, за это время сигнал $>$ передастся вправо и окажется во второй ячейке, таким образом, будет обеспечена синхронизация. В качестве сигнала, бегушего влево, используем уже имеющееся образование из 1 во втором слое и / в шестом слое.

Если $c(x, y)=>, a(x+1, y)=\Pi$, то $b^{\prime}(x, y)=, c^{\prime}(x, y)=\Pi, f^{\prime}(x, y)=/$.

Далее им нужно разрешить двигаться по массиву из нулей и единиц первого слоя.

Если $a(x, y)=1$ или $a(x, y)=0, b(x+1, y)=1, f(x+1, y)=1$, то $b^{\prime}(x, y)=1$, $f^{\prime}(x, y)=/$.

При подходе к левому краю кода они формируют сигнал $>$.

Если $a(x-1, y)=\Pi, a(x, y) \neq \Pi, b(x, y)=1, f(x, y)=/$, то $c^{\prime}(x, y)=>$.

Опишем правила движения сигналов пятого слоя $r$ и $R$.

Если $e(x, y)=r$, то $e^{\prime}(x, y)=\Pi$.

Если $e(x-1, y)=r, a(x, y) \neq \Pi$, то $e^{\prime}(x, y)=R$. 
Если $e(x, y)=R$, то $e^{\prime}(x, y)=r$.

При подходе к правому краю $r$ генерирует сигнал $<$.

Если $e(x, y)=r, a(x+1, y)=\Pi$, то $c^{\prime}(x, y)=<, e^{\prime}(x, y)=\Pi$.

Обработка случаев $N=2$ и $N=3$. Наконец, еще одно соображение. Дело в том, что в область применимости ОС $\sigma_{1}$ не входили числа 2 и 3 . Опишем эти исключительные случаи.

В случае $N=2$, если $a(x-1, y)=>, a(x, y)=<, b(x+1, y)=\Pi, e(x+1, y)=\Pi$, $f(x+1, y)=\Pi$, то $a^{\prime}(x, y)=\Pi, b^{\prime}(x, y)=\Pi, c^{\prime}(x, y)=P, e^{\prime}(x, y)=\Pi, f^{\prime}(x, y)=\Pi$.

В случае $N=3$, если $a(x-1, y)=\Pi, b(x-1, y)=\Pi, c(x-1, y)=\Pi, e(x-1, y)=\#$, $f(x-1, y)=\Pi, a(x, y)=1, b(x, y)=\Pi, c(x, y)=>, d(x, y)=\Pi, e(x, y)=r$, $f(x, y)=\Pi, a(x+1, y)=\Pi, b(x+1, y)=\Pi, c(x+1, y)=\Pi, e(x+1, y)=\Pi$, $f(x+1, y)=\Pi$, то $a^{\prime}(x, y)=\Pi, b^{\prime}(x, y)=\Pi, c^{\prime}(x, y)=P, e^{\prime}(x, y)=\Pi, f^{\prime}(x, y)=\Pi$.

Далее символ $P$ двигается вправо, пока не выйдет на границу.

Если $c(x-1, y)=P, a(x, y) \neq \Pi$, то $a^{\prime}(x, y)=\Pi, c^{\prime}(x, y)=c(x-1, y)$.

Описание алгоритма закончено.

Отметим, что функщия переходов ОС была представлена в виде цепочки условий и следствий. Эта цепочка получена автоматически по программе ОС, написанной на некотором языке. Программа, а следовательно, и цепочка условий, имеет некоторые особенности. Именно, она выполняется последовательно сверху вниз, то есть, одновременно могут быть выполнены несколько условий, причем, они могут, как дополнять, так и противоречить друг другу. В случае противоречия, в действительности, выполняется самое последнее условие.

Приведем основные характеристики ОС $\sigma_{2}$. Начнем с числа состояний ячейки. Число состояний ОС $\sigma_{1}$, естественно, сохраняется, и к нему еще добавляются 27 новых состояний, возникших при трансформировании кода.

Далее найдем размер максимальной конфигурации. В воспользуемся результатом для OC $\sigma_{1}$. Тогда для ОС $\sigma_{2}$ будет иметь место оценка $\max \left\{n^{3} / 4+5 n^{2} / 4+2 n, N\right\}$, где, понятно, $n=[\log N]+1$. Легко видеть, что почти для всех $N$ максимальной конфигурацией будет начальная.

Оценим время работы ОС $\sigma_{2}$. Ясно, что в случае нечетного $N$ оно будет складываться из времени, затраченного на преобразование палочного кода в двоичный, и времени функщионирования ОС $\sigma_{1}$. Посчитаем, чему равно первое слагаемое.

Формирование сигнала \# происходит на $[N / 2]+1$ такте. Для копирования кода из второго слоя в первый надо $2[\log N]-1$ тактов. На достижение сигналом $r$ правой части кода и постановку им символа $<$ необходимо $2[\log N]+1$ тактов. Всего на перекодирование надо потратить $[N / 2]+4[\log N]+1=[N / 2]+4 n-3$ тактов.

Таким образом, для нечетного $N$ время работы ОС $\sigma_{2}$ будет меньше, чем $N / 2+2 \sqrt{N}+n^{2} / 2+9 n+4$. Для четных $N$ время работы еще меньше, оно ограничено сверху числом $N / 2+n$.

Итак, ОС $\sigma_{2}$ полностью описана, тем самым, лемма 3 доказана.

\section{5. Основная теорема и следствие}

Утверждение основной теоремы следует из лемм 1, 2 и 3.

Если проследить построение ОС $\sigma_{2}$, то можно заметить, что при перекодировке натурального числа из палочного в двоичное представление нигде не используется тот факт, что $\mathrm{OC} \sigma_{2}-$ плоская однородная структура, то есть линейная однородная структура 
с шаблоном соседства $\{-1,0,1\}$ и с функцией перехода, полученной из уже описанной функщии переходов для ОС $\sigma_{2}$ выбрасыванием несущественных переменных, будет также осуществлять перекодировку. Далее, лемма 2 верна и для линейных ОС. Теперь можно по аналогии рассмотреть задачу проверки простоты натурального числа в классе линейных однородных структур. Обозначим $L(N)$ функцию временной сложности для этой задачи.

Следствие 1. При $N \rightarrow \infty$

$$
L(N) \sim N / 2 .
$$

Автор благодарен В. Б. Кудрявцеву за постановку задачи и ценные советы по ее решению.

\section{Список литературы}

1. Кудрявцев В. Б., Алешин С. В., Подколзин А. С., Введеиие в теорию автоматов. Наука, Москва, 1985.

2. Кудрявцев В. Б., Подколзин А. С., Болотов А. А., Осиовы теории одиородных структур. Наука, Москва, 1990.

3. Василенко О. Н., Современные способы проверки простоты чисел. Обзор. Кибери. сб. 25, 162188.

Статья поступила 11.10.2002. 